\title{
Authors' reply to Lachenmeier and Rehm
}

\author{
Martin McKee professor of European public health, Roza Adany professor of public health, David \\ A Leon professor of epidemiology
}

London School of Hygiene and Tropical Medicine, London WC1H 9SH, UK

We were surprised by Lachenmeier and Rehm's response to our editorial. ${ }^{12}$ Of course efforts should focus primarily on legally sold alcohol, and we have recently published a systematic review to inform such policies. ${ }^{3}$

However, the World Health Organization's quoted estimates for unrecorded consumption are now several years old. More recent data from the UK government suggest a growth in the scale of the problem, and we think it would be complacent not to flag up the potential public health consequences, which may not be trivial in some sections of the community.

The question of beer that the authors raise was not mentioned by us, and we agree that it is not a concern. However, we presume that they do accept that illegally produced spirits are less likely to be subject to the quality standards applied in commercial production. We made it clear that these products can contain a range of toxic alcohols, not that they will always do so, and discussed the circumstances in which this was more likely. We are fully aware that manufacturing standards and toxicological thresholds vary, but we did not feel it necessary to include this detail in a short editorial.

Competing interests: None declared.

1 McKee M, Adany R, Leon DA. Illegally produced alcohol. BMJ 2012;344:e1146. (21 February.)

2 Lachenmeier DW, Rehm J. Perhaps not such a great threat to public health in the UK. BMJ 2012;344:e2251.

3 Bryden A, Roberts B, McKee M, Petticrew M. A systematic review of the influence on alcohol use of community level availability and marketing of alcohol. Health Place 2012;18:349-57. 